\title{
Concerted responses between the chitin-binding protein secreting Streptomyces olivaceoviridis and Aspergillus proliferans
}

\author{
Krzysztof W. Siemieniewicz† and Hildgund Schrempf \\ FB Biologie/Chemie, Universität Osnabrück, Barbarastr. 13, D-49069 Osnabrück, Germany
}

\begin{abstract}
Correspondence
Hildgund Schrempf

schrempf@biologie.

uni-osnabrueck.de
\end{abstract}

Received 9 August 2006

Revised 27 October 2006

Accepted 30 October 2006

\begin{abstract}
Streptomycetes belong to the ecologically important bacterial population within soil, which is also inhabited by many fungi. The highly chitinolytic Streptomyces olivaceoviridis and the ascomycete Aspergillus proliferans were chosen as models to test for interactions among bacteria and fungi. In medium lacking a soluble carbon source, individually cultivated spores of the bacterium $S$. olivaceoviridis and the fungus $A$. proliferans do not germinate. However, as shown by viability tests, cultivation of a mixture of both spore types provokes successive events: (i) stimulation of the germination of S. olivaceoviridis spores, (ii) initiation of the outgrowth of some fungal spores to which the S. olivaceoviridis chitinase ChiO1 adheres, (iii) massive extension of viable networks of $S$. olivaceoviridis hyphae at the expense of fungal hyphae and (iv) balanced proliferation of closely interacting fungal and S. olivaceoviridis hyphae. The replacement of the $S$. olivaceoviridis wild-type strain by a chromosomal disruption mutant $(\Delta C)$, lacking production of the extracellular chitinbinding protein $\mathrm{CHB} 1$ but still secreting the chitinase ChiO1, provokes (v) germination of each spore type, (vi) retarded development of both partners, followed by (vii) preferential proliferation of the fungus. Together with biochemical and immunomicroscopy studies, the data support the conclusion that CHB1 molecules aggregate to an extracellular matrix, maintaining a close contact, followed by several concerted responses of the bacterium and the fungus.
\end{abstract}

\section{INTRODUCTION}

Streptomycetes efficiently utilize chitin as a sole carbon as well as a nitrogen source (Kutzner, 1981). The features of the highly chitinolytic Streptomyces olivaceoviridis have been investigated in detail (Beyer \& Diekmann, 1985). This species secretes the chitin-binding protein $\mathrm{CHB} 1$ that targets $\alpha$-chitin, whose linear chains of $\beta$-glycosidic linked $N$ acetylglucosamine (NAG) residues are ordered in an antiparallel arrangement (Schnellmann et al., 1994; Zeltins \& Schrempf, 1995). The strain possesses a set of chitinases, of which the exochitinase ChiO1 has been analysed in detail (Blaak et al., 1993; Blaak \& Schrempf, 1995). The enzyme consists of a catalytic domain, a linker region and a binding domain that can target a range of chitin types, including those within hyphae of fungi. ChiO1 degrades chitin predominantly to $N, N^{\prime}$-diacetylchitobiose (chitobiose) (Blaak \& Schrempf, 1995). This disaccharide, as well as NAG, was identified to be taken up via the newly discovered

†Present address: Dept of Haematology, University of Cambridge, Long Road, Cambridge CB2 2PT, UK.

Abbreviations: CHB1, chitin-binding protein; chb1, gene for chitinbinding protein; $\Delta \mathrm{C}$, mutant lacking the chb1 gene; $\mathrm{ChiO1}$, chitinase; $N A G, N$-acetylglucosamine; $t s r$, gene for thiostrepton resistance; TEM, transmission electron microscopy; WT, wild-type.
$\mathrm{ABC}$ transporter $\mathrm{Ngc}$ (Xiao et al., 2002). In addition, the strain has a phosphoenolpyruvate-dependent transport system (PTS) for NAG (Wang et al., 2002). Recently the regulation of a corresponding PTS has been explored in Streptomyces coelicolor (Rigali et al., 2006). Streptomycetes are Gram-positive bacteria with a highly complex growth cycle. Upon addition of nutrients, spores germinate and the outgrowing tubes extend to long hyphae that form the substrate mycelium. The depletion of nutrients induces the formation of aerial hyphae, in which spores develop. In addition to chitinases, streptomycetes produce many other enzymes (cellulases, amylases, proteases, lipases) that are important for the turnover of macromolecules as well as for humus formation (Kutzner, 1981). The dominant natural habitat of streptomycetes is the soil. Among many other organisms, they encounter here fungi, which play a central role in litter decomposition and biogeochemical cycling (Klein \& Paschke, 2004).

The fungal kingdom is very diverse. While plant-associated fungi are often discussed (reviewed by Harrison, 2005), the role of free-living filamentous ones has been little investigated.

Aspergillus species belong to the ascomycetes - filamentous fungi that build an extended network of vegetative hyphae 
and can form spores that are able to withstand harsh environmental conditions. Aspergillus hyphae have a complex cell wall which comprises chitin, glucan, mannan and proteins (Adams, 2004).

In this report, we show that in co-cultures Aspergillus proliferans and S. olivaceoviridis form a closely tangled coexisting network of fungal and streptomycete hyphae. Immunological studies, including a designed S. olivaceoviridis mutant strain lacking a functional chbl gene for the chitin-binding protein $\mathrm{CHB} 1$, revealed the importance of the chitin-binding protein for the close and balanced interaction of the bacterial and the fungal hyphae.

\section{METHODS}

Strains. Streptomyces olivaceoviridis has been described (Beyer \& Diekmann, 1985). The S. olivaceoviridis mutant $\Delta \mathrm{C}$ carries a substitution of the major portion of the chbl gene (Schnellmann et al., 1994; Zeltins \& Schrempf, 1997) by a thiostrepton resistance gene (tsr) and was kindly provided by A. Zeltins from our group. Aspergillus proliferans was purchased from the DSM (Braunschweig, Germany). E. coli M15[pREP4] (Qiagen) contains the construct pBA1, consisting of the chb1 gene cloned in the pQ32 vector (Svergun et al., 2000).

Cultivation of strains. The S. olivaceoviridis wild-type (WT) and $\Delta \mathrm{C}$ mutant were grown on agar plates containing complete medium (Schnellmann et al., 1994) until sporulation occurred. A. proliferans was grown on agar plates containing yeast extract medium (Vionis et al., 1996) until spores were formed. The spores were removed with sterile water, filtered through cotton, and counted. The spore suspensions of the $S$. olivaceoviridis WT, the $\Delta \mathrm{C}$ mutant $\left(2.5 \times 10^{9}\right.$ spores $\left.\mathrm{ml}^{-1}\right)$ and $A$. proliferans $\left(2.5 \times 10^{8}\right.$ spores $\left.\mathrm{ml}^{-1}\right)$ were stored at $-20{ }^{\circ} \mathrm{C}$ in $40 \%$ glycerol. Having washed out residual glycerol, spores of each $S$. olivaceoviridis strain $\left(5 \times 10^{6}\right)$ were inoculated separately in a flask $(100 \mathrm{ml})$ containing $5 \mathrm{ml}$ minimal medium (Hopwood et al., 1985) supplemented with $0.5 \% \mathrm{NAG}$, or without carbon source (control). Alternatively, the spores of $A$. proliferans $\left(1 \times 10^{6}\right)$ were mixed with 10 -fold excess of those from the S. olivaceoviridis WT or the $\Delta \mathrm{C}$ strain. A. proliferans spores inoculated in medium without carbon source served as control. Cultivation was done at $30{ }^{\circ} \mathrm{C}$ without shaking (to avoid pellet formation). Samples were taken at the indicated time intervals (see Results) and subjected to microscopy. E. coli M15[pREP4]/pBA1 (Svergun et al., 2000) was grown in LB medium (Sambrook et al., 1989).

Microscopic investigations and viability tests. Portions of the cultures were inspected under visible light (phase-contrast) at various magnifications. In parallel, the samples were subjected to a viability assay (Fernandez \& Sanchez, 2002) and they were subsequently examined under UV using filter sets (Zeiss) for FITC (excitation, HQ 480/40; beam splitter, Q 505 LP; emission, HQ 535/50) and Texas red (HQ 560/55, Q 595 LP, HQ 645/75, respectively). Living cells exhibited green fluorescence, whereas dead mycelia were seen in red.

Immunological studies. Antibodies against $\mathrm{CHB} 1$ had been raised previously (Schnellmann et al., 1994). To analyse the presence of CHB1 associated with the fungal hyphae, aliquots of the mycelia obtained during co-culture of A. proliferans and S. olivaceoviridis WT or $\Delta \mathrm{C}$ mutant strain were pre-treated with $2 \%$ BSA in PBS and incubated with anti-CHB1 antibodies (diluted 1:200) for $60 \mathrm{~min}$. Secondary Alexa Fluor 647-labelled antibodies (diluted $1: 200$ ) were purchased from Molecular Probes. Samples were inspected under both phase-contrast and UV using a filter set (Zeiss) for Cy5 (excitation, HQ 620/60; beam splitter, Q 660 LP; emission, HQ 700/75).

To test the presence of extracellular CHB1, the culture filtrate was precipitated with $\left(\mathrm{NH}_{4}\right)_{2} \mathrm{SO}_{4}(90 \%$ saturation). Aliquots of the resuspended proteins were separated in $12.5 \%$ polyacrylamide gels containing $0.1 \%$ SDS, transferred onto a PVDF membrane (Pall Europe), and treated with anti-CHB1 antibodies and secondary antibodies, as described earlier (Zeltins \& Schrempf, 1997).

Test for the presence of chitinase. Using p-nitrophenylchitobiose as substrate, chitinase activity was tested as described earlier (Blaak et al., 1993; Blaak \& Schrempf, 1995). To determine the presence of the chitinase $\mathrm{ChiO} 1,10 \mu \mathrm{l}$ portions were spotted onto a nylon membrane and tested for immunoreaction with specific antibodies (raised previously in rabbit). To test for associated chitinase, a $50 \mu \mathrm{l}$ portion of each culture was dropped onto a slide (pre-treated with polylysine: Schnellmann et al., 1994; Zeltins \& Schrempf, 1995), and incubated for $1 \mathrm{~h}$ in the presence of $2 \%$ BSA in PBS followed by $1.5 \mathrm{~h}$ with anti-chitinase antibodies (diluted $1: 200$ in $2 \%$ BSA in PBS). After 10 washes with PBS, samples were treated for $1 \mathrm{~h}$ with $1: 300$ diluted secondary anti-rabbit antibodies (labelled with Cy5, Molecular Probes). Finally, slides were washed 10 times with PBS and inspected by microscopy under phase-contrast and UV (with the Zeiss filter set for Cy5; see above for specifications).

Aggregation properties of CHB1. E. coli[pREP4]/pBA1 (Svergun et al., 2000) was grown in LB medium (Sambrook et al., 1989) and induced in the exponential phase $\left(\mathrm{OD}_{600}\right.$ 0.6) with IPTG $\left(1 \mathrm{mmol} \mathrm{ml}{ }^{-1}\right)$ for $90 \mathrm{~min}$. Cells were harvested and the His-tagged $\mathrm{CHB} 1$ protein was released by osmotic shock treatment as described earlier for an E. coli strain containing the same vector with the $c h b B$ gene (Chu et al., 2001). The washed cells were suspended in $30 \mathrm{mmol} \mathrm{Tris} / \mathrm{HCl} \mathrm{ml}{ }^{-1}, \mathrm{pH} 8,20 \%(\mathrm{w} / \mathrm{v})$ sucrose, incubated on ice for $10 \mathrm{~min}$, sedimented $\left(8000 \mathrm{~g}, 15 \mathrm{~min}, 4^{\circ} \mathrm{C}\right)$, resuspended in ice-cold $\mathrm{MgSO}_{4}\left(5 \mathrm{mmol} \mathrm{m}^{-1}\right)$ for $10 \mathrm{~min}$ and centrifuged for $15 \mathrm{~min}$ at $4{ }^{\circ} \mathrm{C}$. The supernatant was collected, equilibrated to $50 \mathrm{mmol} \mathrm{Na} \mathrm{HPO}_{4} \mathrm{ml}^{-1}, \mathrm{pH} 8,300 \mathrm{mmol} \mathrm{NaCl} \mathrm{ml}{ }^{-1}, 10 \mathrm{mmol}$ imidazole $\mathrm{ml}^{-1}$, and applied to Ni-NTA resin (Qiagen). The protein was subsequently purified by affinity and ion-exchange chromatography and subjected to gel filtration on a Superdex 75 column (HR 10/30, Amersham Biosciences), equilibrated with $20 \mathrm{mmol}$ Tris/ $\mathrm{HCl}$ $\mathrm{ml}^{-1}$ ( $\mathrm{pH} 7.0$ ) containing $0.1 \mathrm{M} \mathrm{NaCl}$. Peak fractions cross-reacting with anti-CHB1 antibodies were separated in $12.5 \%$ polyacrylamide gels containing $0.1 \%$ SDS and stained with Coomassie brilliant blue R250 (Laemmli, 1970).

Electron microscopy. Gold labelling of CHB1 was based on the instructions of the colloidal gold manufacturer (British BioCell International). After overnight dialysis against $2 \mathrm{mmol}$ borax $\mathrm{ml}^{-1}$ $\mathrm{pH} 8.6$ (corresponds to the $\mathrm{pI}$ value of CHB1), one part of the protein solution $\left(0.1 \mathrm{mg} \mathrm{ml}^{-1}\right)$ was mixed dropwise with 18.75 parts of the colloidal gold (diameter $10 \mathrm{~nm}$ ) suspension that had been adjusted to $\mathrm{pH} 8.6$ with $\mathrm{K}_{2} \mathrm{CO}_{3}\left(100 \mathrm{mmol} \mathrm{ml}^{-1}\right)$. Following a $5 \mathrm{~min}$ incubation at room temperature, BSA $(10 \% \mathrm{w} / \mathrm{v})$ was added to the final concentration of $0.9 \%$ and the solution was agitated for $10 \mathrm{~min}$. After centrifugation $\left(25000 \mathrm{~g}, 60 \mathrm{~min}\right.$ at $\left.4{ }^{\circ} \mathrm{C}\right)$ the supernatant containing the unlabelled CHB1 was removed and the pellet comprising gold-labelled CHB1 was washed with $100 \mathrm{mmol}$ Tris/ $\mathrm{HCl} \mathrm{ml}{ }^{-1} \mathrm{pH} 8.2$ buffer containing BSA (1\%) and $\mathrm{NaN}_{3}(0.1 \%)$, centrifuged and suspended in the same buffer. Aliquots $(10 \mu \mathrm{l})$ were placed on carbon-coated copper grids (300 mesh), treated with uranyl acetate (Leal-Morales et al., 1988), and inspected by transmission electron microscopy (TEM) with an EM9 microscope (Zeiss). For immunodetection, aliquots $(10 \mu \mathrm{l})$ of freshly isolated CHB1 were placed on the grids. After saturation with PBS pH 8.3 containing BSA $(2 \%)$, the grids were incubated with anti-CHB1 antibodies 
and, subsequently, with gold-labelled secondary antibodies (SigmaAldrich). The grids were contrasted with uranyl acetate and inspected by TEM.

\section{RESULTS}

\section{Features of S. olivaceoviridis WT and the $\Delta C$ mutant}

The S. olivaceoviridis WT secretes the chitin-binding protein $\mathrm{CHB} 1$ in the presence of chitin, which promotes an efficient transcription of the chb1 gene (Schnellmann et al., 1994). The chromosomal DNA of the designed S. olivaceoviridis disruption mutant strain $\Delta \mathrm{C}$ (see Methods) contains the $t s r$ gene in place of the major portion of the chb1 gene.

The spores of the $\Delta \mathrm{C}$ mutant and the WT strain germinated equally well in minimal medium containing low-molecularmass carbon sources (NAG, sucrose, glucose or glycerol). Many short germ tubes outgrew within $4 \mathrm{~h}$ and often formed fine networks that rapidly (within $10 \mathrm{~h}$ ) expanded to build substrate mycelia, as exemplified for the WT strain (Fig. 1a, b) and the $\Delta \mathrm{C}$ mutant (Fig. 1d, e) growing in minimal medium supplemented with NAG.
In contrast to the S. olivaceoviridis strains (Fig. 1a, b, d, e), A. proliferans cannot utilize NAG hence its spores cannot germinate (Fig. 1g, h, white arrows). In control medium lacking NAG, none of the S. olivaceoviridis spores (Fig. 1c, f, black arrows) nor those of $A$. proliferans (Fig. 1i, white arrow) germinated (data presented for $22 \mathrm{~h}$ ).

\section{Responses of S. olivaceoviridis WT and $\boldsymbol{A}$. proliferans during co-cultivation}

For further studies, spores of each S. olivaceoviridis strain were co-cultured with those of the fungus in minimal medium without soluble carbon source. The spores of $S$. olivaceoviridis WT germinated within $4 \mathrm{~h}$ and the tiny hyphae formed a tangled network (Fig. 2a), closely associating with the clusters of $A$. proliferans spores (white arrow in Fig. 2a). During prolonged incubation (up to $10 \mathrm{~h}$ ), the network of the S. olivaceoviridis WT hyphae extended considerably (Fig. 2b) among large clusters of many fungal spores (white arrow in Fig. 2b).

Extension of the incubation time to $18 \mathrm{~h}$ mainly led to further proliferation of the $S$. olivaceoviridis hyphae, which formed a myceliar network (Fig. 3a). Portions of co-cultures
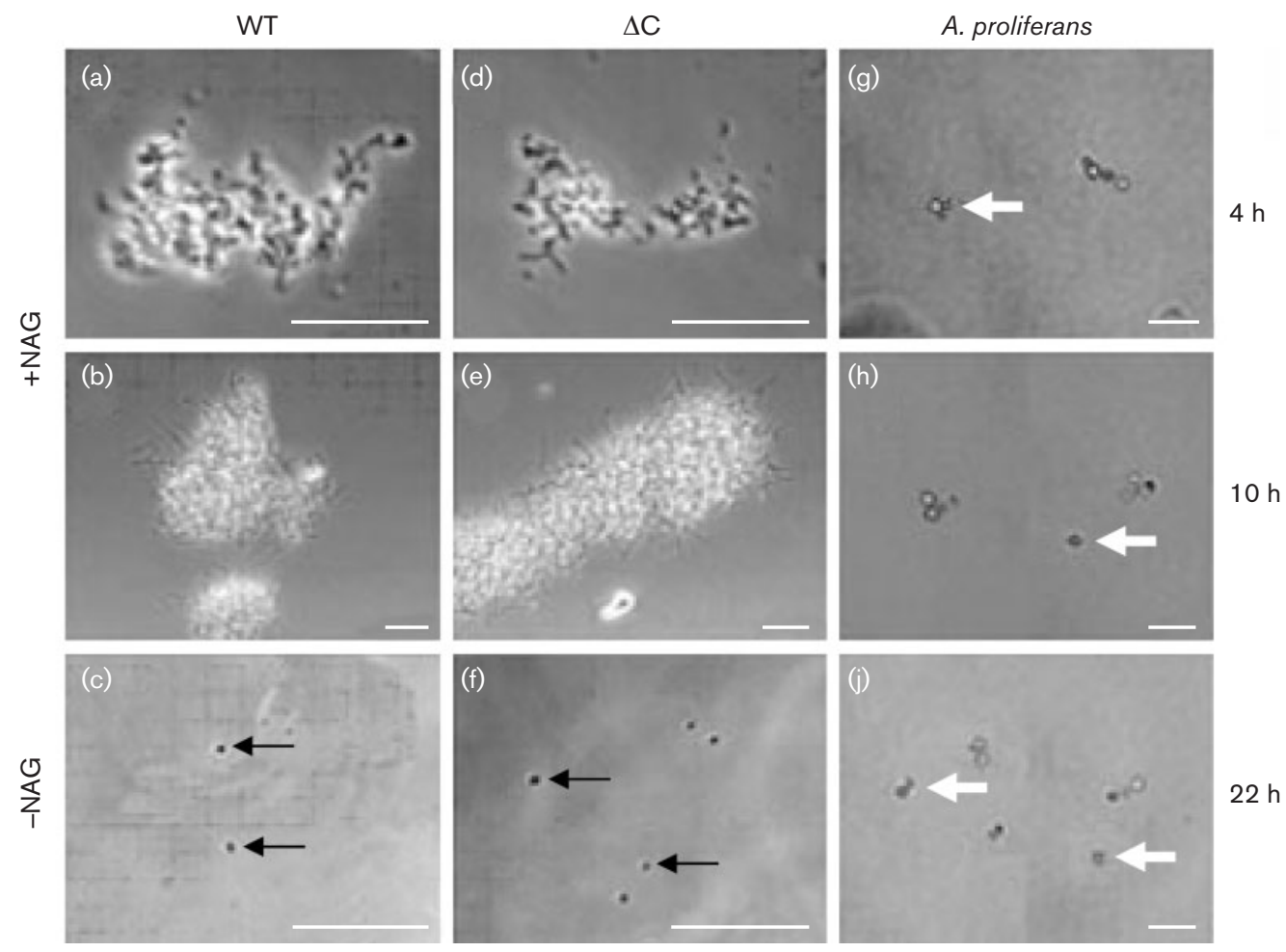

Fig. 1. Growth characteristics of the strains. Spores of $S$. olivaceoviridis WT (a, b, c), the $\Delta \mathrm{C}$ mutant strain (d, e, $\mathrm{f}$ ) or $A$. proliferans $(\mathrm{g}, \mathrm{h}, \mathrm{i})$ were incubated without shaking at $30^{\circ} \mathrm{C}$ in minimal medium supplemented with $0.5 \%$ NAG for $4 \mathrm{~h}$ (a, $\mathrm{d}, \mathrm{g}$ ) or for $10 \mathrm{~h}(\mathrm{~b}, \mathrm{e}, \mathrm{h})$. As a control, spores of each strain were incubated in medium without NAG, for up to $22 \mathrm{~h}$. Germination did not occur, even after $22 \mathrm{~h}$ of incubation (c, $\mathrm{f}, \mathrm{i}$ ). Spores of $\mathrm{S}$. olivaceoviridis (black arrows) and A. proliferans (white arrows) are marked. Bars, $25 \mu \mathrm{m}$. 


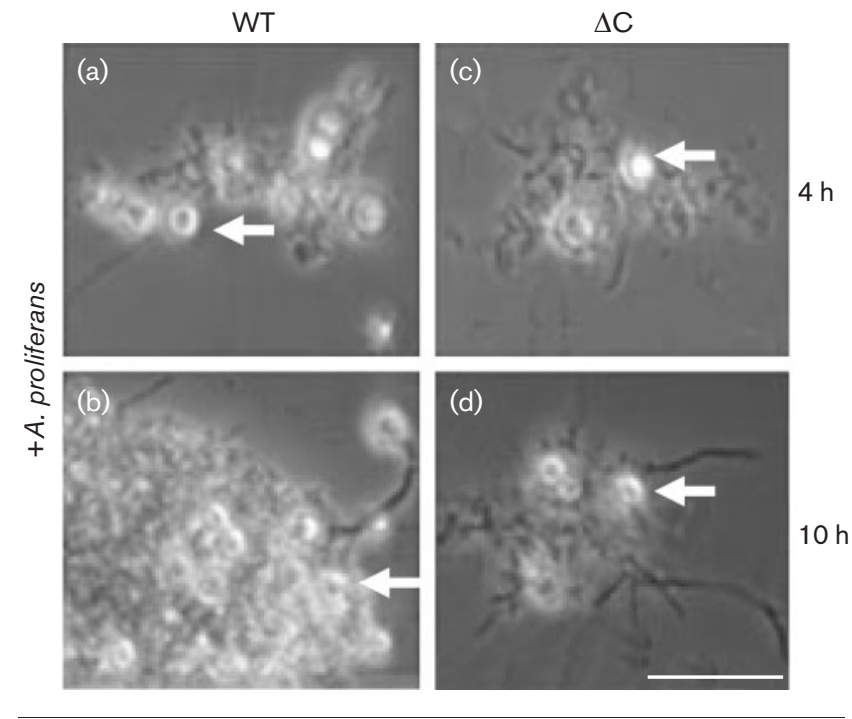

Fig. 2. Spores of $A$. proliferans $\left(1 \times 10^{6}\right)$ were inoculated in $5 \mathrm{ml}$ minimal medium without carbon source together with spores $\left(1 \times 10^{7}\right)$ of $S$. olivaceoviridis WT $(a, b)$ or together with the $\Delta \mathrm{C}$ mutant (c, d) and kept without shaking at $30^{\circ} \mathrm{C}$. Aliquots were inspected after 4 and $10 \mathrm{~h}$ by phase-contrast microscopy. A. proliferans spores (white arrows) are marked. Bar, $25 \mu \mathrm{m}$.

were additionally subjected to a viability test. The test had previously been established for bacteria but had recently also been applied to streptomyces hyphae (Fernandez \& Sanchez, 2002). This procedure involves differential staining of damaged (dead) cells with propidium iodide, which can only enter damaged (dead) cells and subsequently intercalate into the DNA, leading to red fluorescence. Living hyphae can take up the dye SYTO 9, which after its interaction with DNA results in green florescence of hyphae under UV. In contrast to the well-growing (Fig. 3a) and viable (green in Fig. 3b) S. olivaceoviridis hyphae, those of the fungi (white arrow in Fig. 3a) were developing slowly up to $18 \mathrm{~h}$ and they often appeared dead in the viability test (red-appearing fungal filament; white arrow in Fig. 3b). Further co-cultivation (up to $22 \mathrm{~h}$ ) led to the emergence of a few more outgrowing fungal hyphae and the S. olivaceoviridis hyphae continued to be mostly viable (green) and extending on to fungal spores and some dead fungal hyphae (Fig. 3c white arrowhead, 3d). Further incubation (up to $34 \mathrm{~h}$ ) led to the extended proliferation of S. olivaceoviridis as well as some more fungal hyphae (Fig. 3e, white arrowhead). Extended regions of $S$. olivaceoviridis hyphae were viable (green in Fig. 3f) and only a few patches were dead (red in Fig. 3f). In contrast, well-developing A. proliferans hyphae (as judged by phase-contrast microscopy) did not exhibit green fluorescence, indicating that they cannot take up the SYTO 9 dye. Consequently, green fluorescence always correlated only with living hyphae of each of the $S$. olivaceoviridis strains.

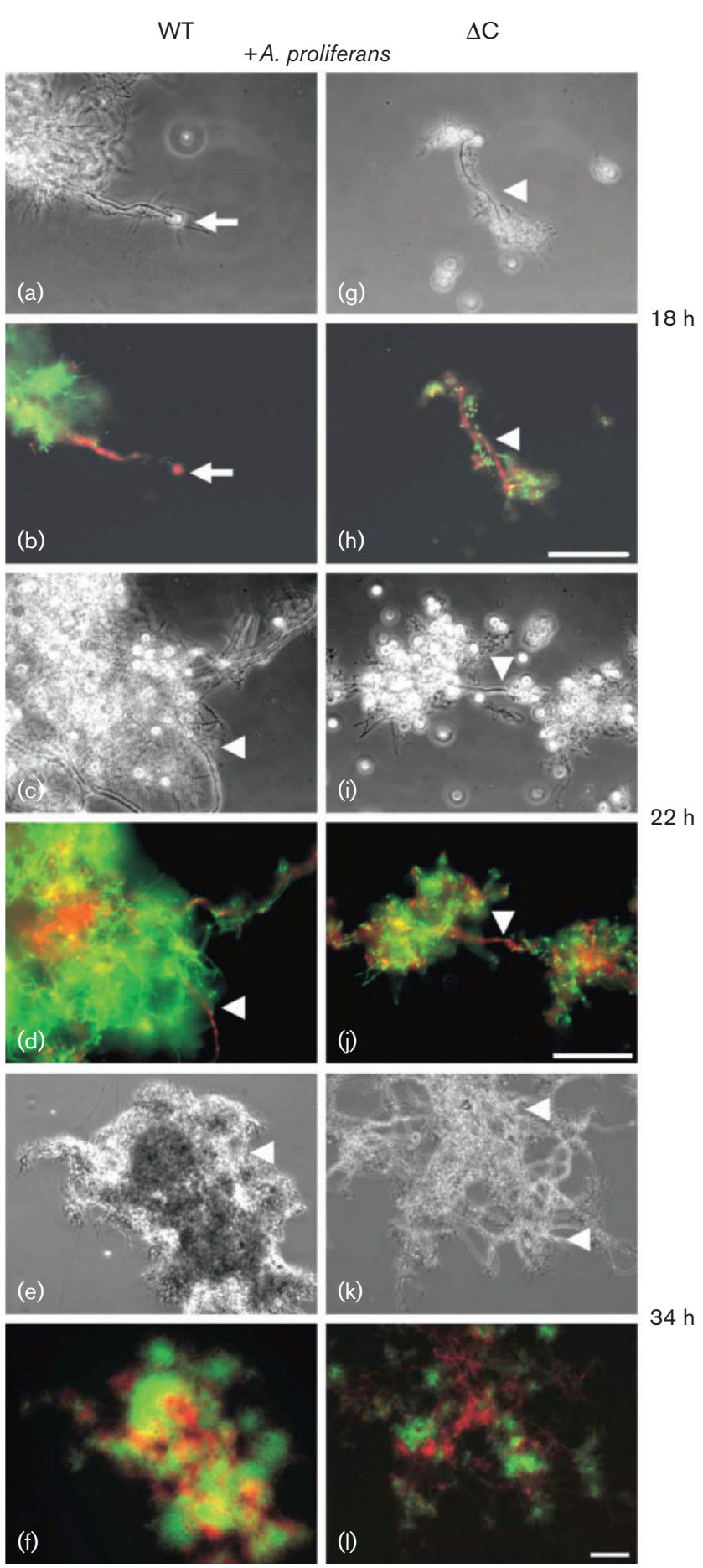

Fig. 3. Viability test of co-cultures. Spores of $S$. olivaceoviridis WT (or the $\Delta \mathrm{C}$ mutant) were incubated together with $A$. proliferans in minimal medium without carbon source, as described in Fig. 2. Having been subjected to a viability test (see Methods), samples of each culture were inspected by phasecontrast under white light ( $a, c, e, g, i, k)$ and UV (b, d, f, h, j, $\mathrm{l}$ ), in the presence of a filter set for FITC or Texas red (see Methods for specifications). Under UV living hyphae appear green, dead ones are red. $A$. proliferans spores (white arrows) and hyphae (white arrowheads) are marked. Bars, $100 \mu \mathrm{m}$. 


\section{Reduced interaction between $A$. proliferans and the $S$. olivaceoviridis $\Delta \mathrm{C}$ mutant}

During early periods $(4-10 \mathrm{~h})$ of co-cultivation the $S$. olivaceoviridis $\Delta \mathrm{C}$ mutant developed slowly on a few fungal spores (white arrows in Fig. $2 \mathrm{c}$ and $\mathrm{d}$ ). After continued incubation $(18 \mathrm{~h})$ the hyphae of the $\Delta \mathrm{C}$ mutant extended (Fig. 3g, i, k). Up to 22 h only a few fungal spores outgrew to hyphae (white arrowheads in Fig. $3 \mathrm{~g}-\mathrm{j}$ ). However, after extension of the co-culture time to $34 \mathrm{~h}$ the network of the fungal hyphae extended considerably (white arrowheads in Fig. 3k), among residual dead (red) patches and a few interspersed living (green) hyphae from the S. olivaceoviridis $\Delta \mathrm{C}$ mutant (Fig. 3j).

If NAG was added to each type of co-cultures, the growth characteristics of each $S$. olivaceoviridis strain were very similar (up to $22 \mathrm{~h}$ ), leading to dominant growth of $S$. olivaceoviridis hyphae (of WT or $\Delta \mathrm{C}$ ) compared to the fungus (Fig. 4). The data indicate that the low-molecularmass carbon source NAG $(0.5 \%)$ is used preferentially in the

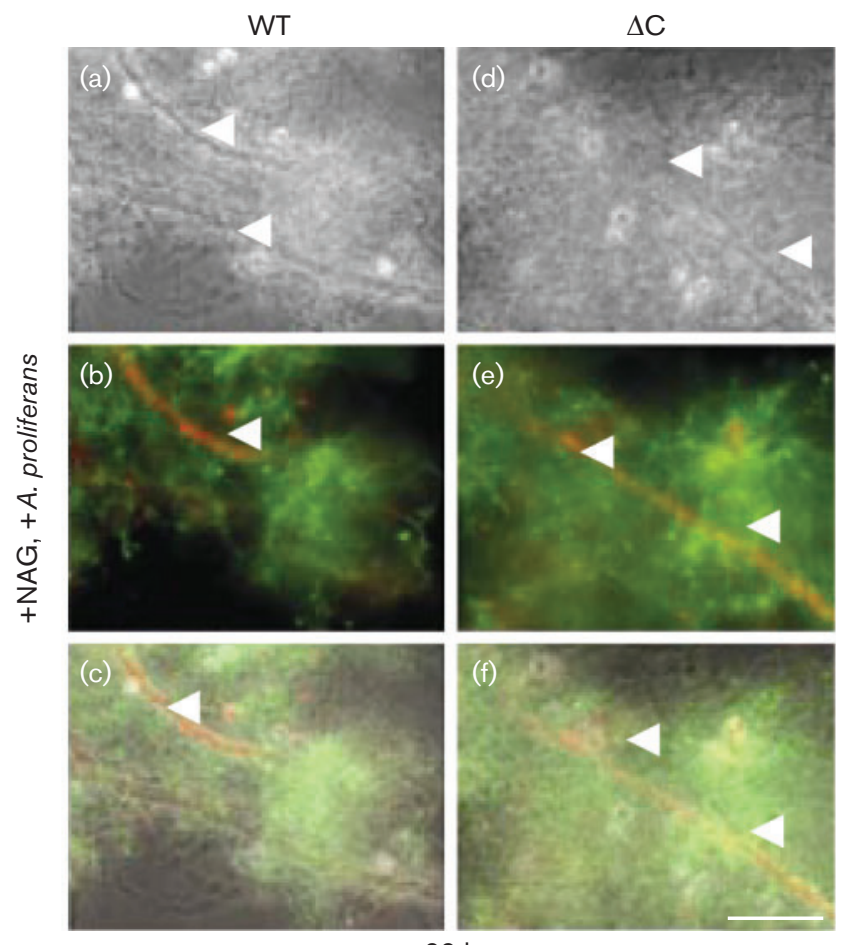

$22 \mathrm{~h}$

Fig. 4. Viability test of co-cultures in the presence of NAG. Spores of $S$. olivaceoviridis WT or $\Delta \mathrm{C}$ were grown with those of $A$. proliferans for $22 \mathrm{~h}$ in minimal medium supplemented with NAG $(0.5 \%)$, as described in Fig. 1. Samples were subjected to a viability test (see Methods) and inspected by light ( $a, d)$ and fluorescence microscopy (b, e) as described in Fig. 3. The merged pictures $a+b$ and $d+e$ resulted in $c$ and $f$, respectively. A. proliferans hyphae (white arrows) are marked. Bar, $25 \mu \mathrm{m}$. additional presence of fungal spores, which cannot germinate with NAG as carbon source (Fig. 1g-i).

\section{Deposition of chitin-binding protein CHB1 within co-cultures}

Antibodies were used to score the distribution of $\mathrm{CHB} 1$ during co-culture of S. olivaceoviridis WT and A. proliferans (Fig. 5a, b). CHB1 levels were found to correlate with the expansion of the network of $S$. olivaceoviridis hyphae in the presence of fungus and the amount of CHB1 was notably higher within the closely interacting networks. Many patches of the deposited CHB1 were immunodetected, primarily in regions containing well-developed fungal hyphae in close vicinity to the $S$. olivaceoviridis hyphae
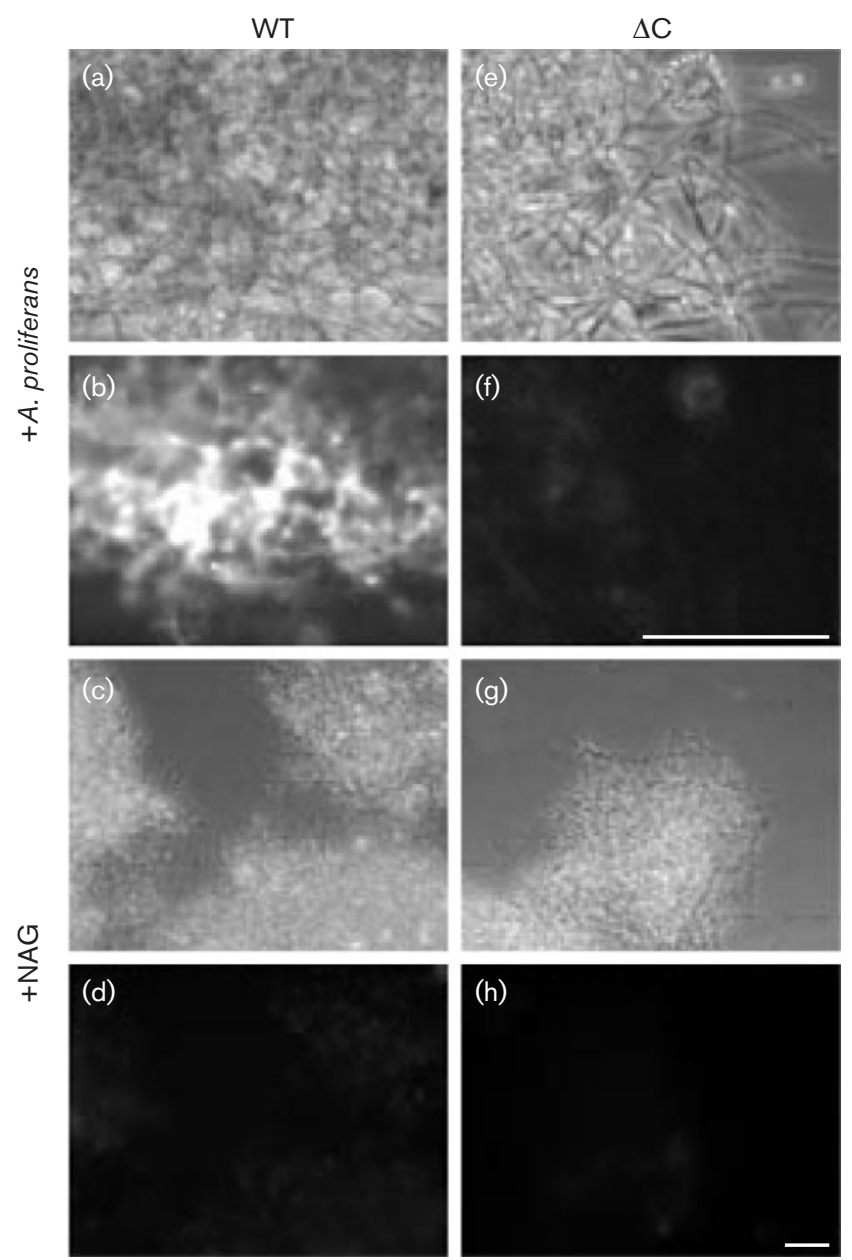

Fig. 5. Immunodetection of the CHB1 protein within the cocultures. S. olivaceoviridis WT $(\mathrm{a}-\mathrm{d})$ or the $\Delta \mathrm{C}$ mutant $(\mathrm{e}-\mathrm{h})$ were grown in minimal medium with NAG $(c, d$ and $g, h)$ or in minimal medium without NAG but as a co-culture with $A$. proliferans ( $\mathrm{a}, \mathrm{b}$ and $\mathrm{e}, \mathrm{f})$. Samples were taken after $63 \mathrm{~h}$, treated with primary anti-CHB1 antibodies and secondary Alexa Fluor 647-labelled antibodies, and inspected under UV (b, d, f, h) in the presence of a filter set for Cy5 (see Methods for specifications). Bars, $25 \mu \mathrm{m}$. 
(Fig. 5b). As expected, CHB1 was not immunodetectable when the S. olivaceoviridis WT strain was replaced by the $\Delta \mathrm{C}$ strain (Fig. 5e, f) or when each of the S. olivaceoviridis strains was cultivated with NAG as a sole carbon source (Fig. $5 c$, d and $g, h$ ).

\section{Aggregation properties of CHB1 molecules}

The purified CHB1 was found to form high-molecular-mass aggregates. Gel filtration (Fig. 6a) and subsequent SDSPAGE analysis (Fig. 6b) after heating $\left(100^{\circ} \mathrm{C}\right)$ revealed a set of differently sized protein bands containing CHB1. Immunological studies (Western blot, not shown) confirmed that these protein bands corresponded to CHB1 monomers, dimers, trimers (traces) and higher oligomers. The aggregation was found to be independent of the treatment with thiol-reducing agents and still occurred in the presence of moderate concentrations of denaturing agents (urea and guanidinium hydrochloride, data not shown), suggesting hydrophobic interactions. The selfaggregation of gold-labelled $\mathrm{CHB} 1$ was visualized by electron microscopy (Fig. 6c). Likewise, patches of highly aggregated CHB1 were immunodetectable with goldlabelled antibodies, in contrast to the control (Fig. 6c).

\section{Synthesis of chitinase in co-cultures}

Chitinase activity was barely detectable in the culture filtrate of each type of co-culture in the early phase (up to $18 \mathrm{~h}$ ) of growth. Immunological studies (dots spotted onto a filter) showed that the previously identified chitinase ChiO1 (Blaak et al., 1993; Blaak \& Schrempf, 1995) was present within culture filtrates. Its amount rose slowly during extension of the cultivation time (up to 3 days) in both types of co-cultures. The resolved protein patterns (SDS-PAGE) of 50-fold concentrated (by ammonium sulphate precipitation) supernatant were identical. Very small (about equal) amounts of a protein (about $55 \mathrm{kDa}$ ) were immunodetectable with anti-ChiO1 antibodies within the supernatant of each type of co-culture (data not shown). This finding prompted us to test for the association of the enzyme. Immunological studies revealed that the chitinase ChiO1 (a)

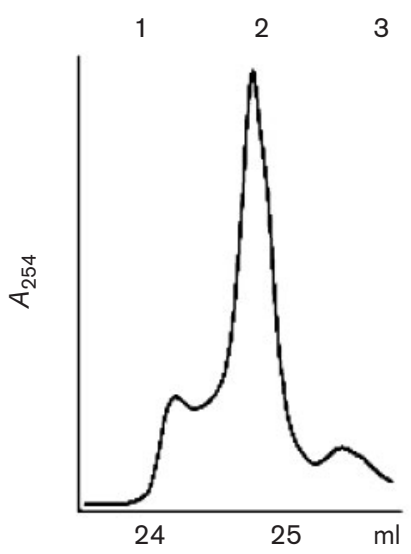

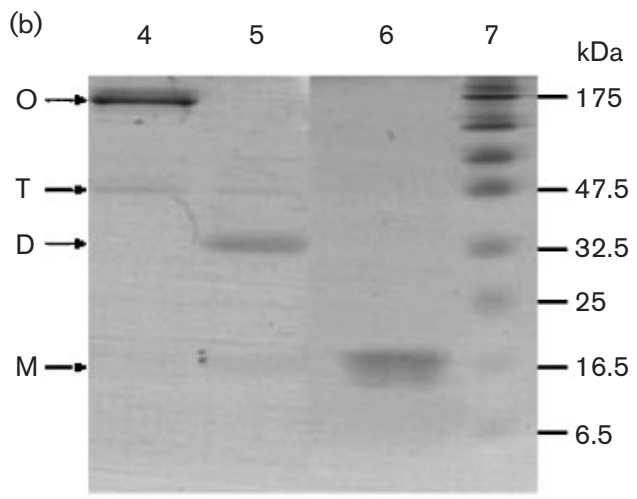

(c)
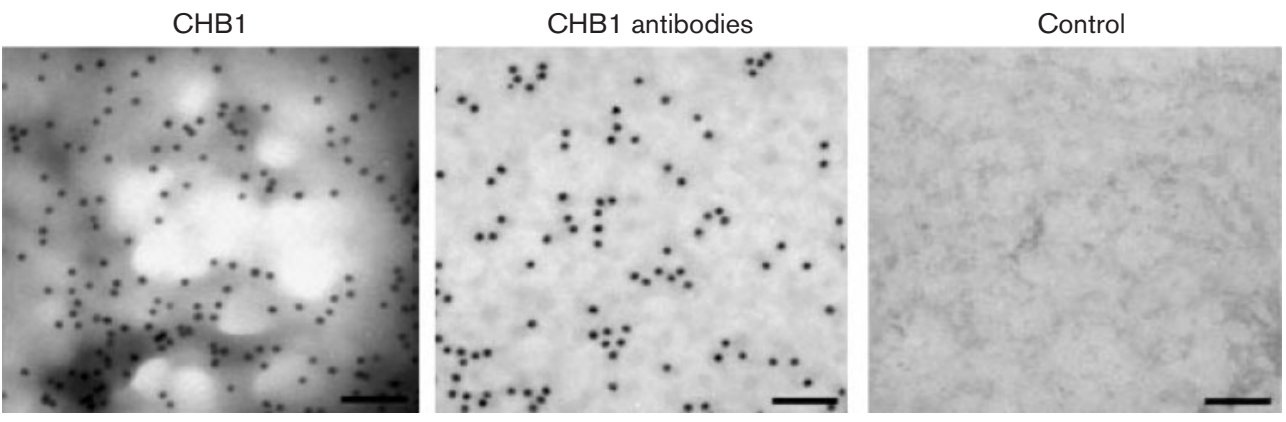

Fig. 6. Analysis of the aggregation properties of CHB1. (a) The purified CHB1 protein was subjected to gel filtration, resulting in the peak fractions 1,2 and 3 . (b) Fraction 1 (aliquot of $30 \mu \mathrm{l}$ ) mainly comprised oligomers $(\mathrm{O})$ and traces of the trimer $(\mathrm{T})$ as shown by SDS-PAGE (lane 4). Fraction $2(10 \mu \mathrm{l})$ contained mainly the dimer (lane 5$)$. In fraction $3(30 \mu \mathrm{l})$ the monomer (M) dominated (lane 6). As a control, reference proteins were analysed (lane 7). (c) Purified CHB1 was goldlabelled, placed on to a copper grid, and inspected directly by TEM (left). A sample of the purified CHB1 was placed on a copper grid, and treated with anti-CHB1 antibodies and secondary gold-labelled antibodies (centre). A sample that contained no $\mathrm{CHB} 1$ served as control (right). Bars, $70 \mathrm{~nm}$. 
was deposited on the fungal spores (white arrows in Fig. 7ad) already during the early phase $(18 \mathrm{~h})$ of cultivation. This effect occurred in both types of co-culture to about equal extent.

\section{DISCUSSION}

Spores of fungi and streptomycetes represent the dormant stage of their life cycle. Our studies showed that in the absence of nutrients individual spores of A. proliferans and the S. olivaceoviridis (WT and $\Delta \mathrm{C}$, respectively) do not form germ tubes. In contrast, the bacterial and fungal spores germinate when mixed in minimal medium lacking a carbon source. In co-culture the spores of $S$. olivaceoviridis WT (Fig. 2a, b) or the $\Delta \mathrm{C}$ mutant (Fig. 2c, d) appear to germinate more rapidly than those of $A$. proliferans (white arrows in Fig. 2). This finding is in agreement with the
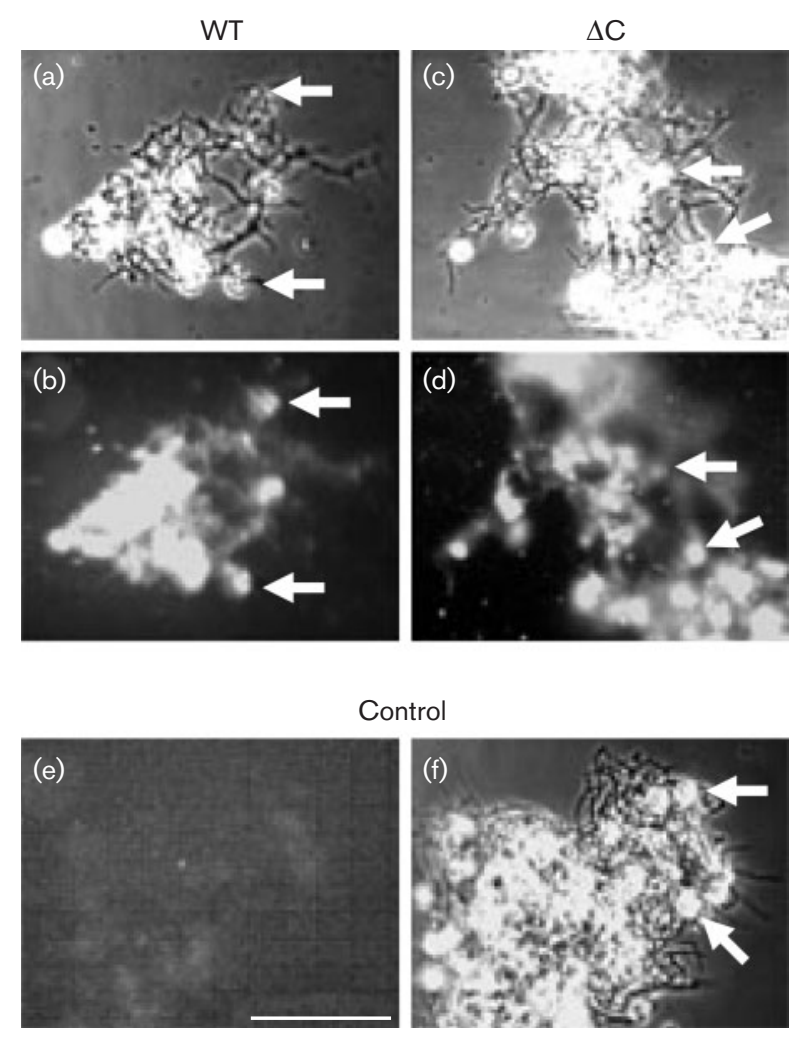

Fig. 7. Detection of chitinase activity. Spores of S. olivaceoviridis WT $(\mathrm{a}, \mathrm{b})$ or the $\Delta \mathrm{C}$ mutant $(\mathrm{c}, \mathrm{d})$ were co-cultivated with those of $A$. proliferans in minimal medium without carbon source for $18 \mathrm{~h}$. Samples were treated with anti-chitinase (ChiO1) antibodies followed by secondary Cy5-labelled antirabbit antibodies and inspected under phase-contrast $(a, c)$ or UV $(b, d)$ in the presence of a filter set for Cy5 (see Methods for specifications). Controls that were done for both types of co-cultures in the absence of secondary antibodies showed the same result; thus they are presented only for $A$. proliferans with the WT strain under phase-contrast (f) and UV (e). A. proliferans spores (white arrows) are marked. Bar, $50 \mu \mathrm{m}$. observation that the culture filtrate from either type of coculture did not enhance the germination of the fungal or the S. olivaceoviridis (WT or $\Delta \mathrm{C}$ ) spores. Knowledge of the molecular mechanism(s) of spore germination in streptomycetes is still scarce. The process is usually observed after exposure to low-molecular-mass nutrients (Kutzner, 1981). In the initial stage unknown signals trigger the influx of water into spores, leading to swelling and subsequently to outgrowth of a germ tube. An S. coelicolor mutant with a non-functional gene for adenylate cyclase was found to have a delay in the germ tube emergence (Susstrunk et al., 1998).

RasA, a small GTP-binding protein, is important for carbon source sensing during Aspergillus nidulans germination. In addition, the cAMP/PKA pathway is believed to be involved. Thus germ tube formation is delayed in the absence of the adenylate cyclase gene (Fillinger et al., 2002). GasC, a member of the group III $\mathrm{G}_{\alpha}$ proteins, appears to control germination but not the carbon sensing in Penicillium marneffei and it has been proposed that GasC signals through the cAMP/PKA pathway (Zuber et al., 2002). Interestingly, serine/threonine and tyrosine kinases, which control cellular responses to environmental stimuli via complex signal transduction networks in eukaryotes, have recently been found in streptomycetes (Umeyama et al., 2002) and their participation in morphogenesis seems likely.

In contrast to S. olivaceoviridis WT, the development of the mutant $\Delta \mathrm{C}$, which does not secrete the chitin-binding protein $\mathrm{CHB} 1$, is considerably retarded during co-culture with A. proliferans (Fig. 2). As visualized by immunomicroscopy, CHB1 forms patches within the network of the $S$. olivaceoviridis WT hyphae interwoven with fungal hyphae and residual fungal spores (Fig. 5a, b). The biochemical and electron microscopy studies revealed that even CHB1 alone forms high-molecular-mass aggregates (Fig. 6), probably via hydrophobic interactions.

During further extension of the co-culture time, the $S$. olivaceoviridis WT strain develops a very well-extended and living mycelium. The fungal hyphae also wrap around the individual or bunched S olivaceoviridis hyphae (Fig. 3a, b). As reported earlier, streptomycetes secrete many extracellular hydrolytic enzymes degrading macromolecules such as glucans, chitin and proteins, which are also located in the cell wall of fungi. Interestingly, the chitinase ChiO1 from $S$. olivaceoviridis was primarily found bound to the fungal spores, suggesting its participation in their degradation. Previous studies have shown that the binding domain of this chitinase mediates its adherence to chitin-containing substrates (Vionis et al., 1996). Therefore, it was not surprising that during early steps of co-cultivation the occurrence of rapidly developing $S$ olivaceoviridis hyphae correlated with the decay of emerging fungal hyphae (Fig. 3b, c, d). Previously, it had been shown that $S$. olivaceoviridis has uptake systems for degradation products such as NAG and chitobiose (Wang et al., 2002; Saito \& Schrempf, 2004). Ultimately, the S. olivaceoviridis WT and 
the fungus co-grow in a balanced and sustainable manner (Fig. 3e).

In co-culture with $A$. proliferans the $S$. olivaceoviridis $\Delta \mathrm{C}$ mutant shows considerably retarded development (Fig. 2c, d) compared to the WT strain (Fig. 2a, b). After $34 \mathrm{~h}$ of coculture the hyphae of $A$. proliferans develop very well and overgrow (Fig. $3 \mathrm{k}$ ) the $\Delta \mathrm{C}$ hyphae, a large portion of which was shown to be no longer alive (Fig. 31). Thus, the fungal hyphae appear to feed well on the decaying $\Delta \mathrm{C}$ strain. Interestingly, various Aspergillus species produce a range of enzymes (Klein \& Paschke, 2004), some of which (e.g. proteases) and antibiotics ( $\beta$-lactams) could contribute to the damage of the emerging bacterial hyphae.

This study is believed to be the first to demonstrate an intimate interaction between a Streptomyces and an Aspergillus strain. Deeper insights into the socio-microbiology of streptomycetes and free-living fungi are of great value for applications and are of high ecological importance.

\section{ACKNOWLEDGEMENTS}

The work was financed in part by a grant of the Deutsche Forschungsgemeinschaft Schr 203/6-3 to H. Schrempf.

\section{REFERENCES}

Adams, D. J. (2004). Fungal cell wall chitinases and glucanases. Microbiology 150, 2029-2035.

Beyer, M. \& Diekmann, H. (1985). The chitinase system of Streptomyces sp. ATCC 11238 and its significance for fungal cell wall degradation. Appl Microbiol Biotechnol 23, 140-146.

Blaak, H. \& Schrempf, H. (1995). Binding and substrate specificities of a Streptomyces olivaceoviridis chitinase in comparison with its proteolytically processed form. Eur J Biochem 229, 132-139.

Blaak, H., Schnellmann, J., Walter, S., Henrissat, B. \& Schrempf, H. (1993). Characteristics of an exochitinase from Streptomyces olivaceoviridis, its corresponding gene, putative protein domains and relationship to other chitinases. Eur J Biochem 214, 659-669.

Chu, H. H., Hoang, V., Hofemeister, J. \& Schrempf, H. (2001). A Bacillus amyloliquefaciens ChbB protein binds $\beta$ - and $\alpha$-chitin and has homologues in related strains. Microbiology 147, 1793-1803.

Fernandez, M. \& Sanchez, J. (2002). Nuclease activities and cell death processes associated with the development of surface cultures of Streptomyces antibioticus ETH 7451. Microbiology 148, 405-412.

Fillinger, S., Chaveroche, M. K., Shimizu, K., Keller, N. \& d'Enfert, C. (2002). cAMP and ras signalling independently control spore germination in the filamentous fungus Aspergillus nidulans. Mol Microbiol 44, 1001-1016.

Harrison, M. J. (2005). Signaling in the arbuscular mycorrhizal symbiosis. Annu Rev Microbiol 59, 19-24.

Hopwood, D. A., Bibb, M. J., Chater, K. F., Kieser, T., Bruton, C. J., Kieser, H. M., Lydiate, D. J., Smith, C. P., Ward, J. M. \& Schrempf, H. (1985). Genetic Manipulation of Streptomyces: a Laboratory Manual. Norwich: John Innes Foundation.

Klein, D. A. \& Paschke, M. W. (2004). Filamentous fungi: the indeterminate lifestyle and microbial ecology. Microb Ecol 47, 224-235.
Kutzner, H. J. (1981). The family Streptomycetaceae. In The Prokaryotes: a Handbook on Habitats, Isolation and Identification of Bacteria, pp. 2028-2090. Edited by M. P. Starr, H. Stolp, H. G. Trüper, A. Balows \& H. Schlegel. Berlin: Springer.

Laemmli, U. K. (1970). Cleavage of structural proteins during the assembly of the head of bacteriophage T4. Nature 227, 680-685.

Leal-Morales, C. A., Bracker, C. E. \& Bartnicki-Garcia, S. (1988), Localization of chitin synthetase in cell-free homogenates of Saccharomyces cerevisiae: chitosomes and plasma membrane. Proc Natl Acad Sci U S A 85, 8516-8520.

Rigali, S., Nothaft, H., Noens, E. E., Schlicht, M., Colson, S., Muller, M., Joris, B., Koerten, H. K., Hopwood, D. A. \& other authors (2006). The sugar phosphotransferase system of Streptomyces coelicolor is regulated by the GntR-family regulator DasR and links $\mathrm{N}$-acetylglucosamine metabolism to the control of development. Mol Microbiol 61, 1237-1251.

Saito, A. \& Schrempf, H. (2004). Mutational analysis of the binding affinity and transport activity for $\mathrm{N}$-acetylglucosamine of the novel $\mathrm{ABC}$ transporter $\mathrm{Ngc}$ in the chitin-degrader Streptomyces olivaceoviridis. Mol Genet Genomics 271, 545-553.

Sambrook, J., Fritsch, E. F. \& Maniatis, T. (1989). Molecular Cloning: a Laboratory Manual, 2nd edn. Cold Spring Harbor, NY: Cold Spring Harbor Laboratory.

Schnellmann, J., Zeltins, A., Blaak, H. \& Schrempf, H. (1994). The novel lectin-like protein CHB1 is encoded by a chitin-inducible Streptomyces olivaceoviridis gene and binds specifically to crystalline $\alpha$-chitin of fungi and other organisms. Mol Microbiol 13, 807-819.

Susstrunk, U., Pidoux, J., Taubert, S., Ullmann, A. \& Thompson, C. J. (1998). Pleiotropic effects of cAMP on germination, antibiotic biosynthesis and morphological development in Streptomyces coelicolor. Mol Microbiol 30, 33-46.

Svergun, D. I., Becirevic, A., Schrempf, H., Koch, M. H. J. \& Grüber, G. (2000). Solution structure and conformational changes of the Streptomyces chitin-binding protein (CHB1). Biochemistry 39, 10677-10683.

Umeyama, T., Lee, P. C. \& Horinouchi, S. (2002). Protein serine/ threonine kinases in signal transduction for secondary metabolism and morphogenesis in Streptomyces. Appl Microbiol Biotechnol 59, 419-425.

Vionis, A., Niemeyer, F., Karagouni, A. D. \& Schrempf, H. (1996). Production and processing of a $59 \mathrm{kDa}$ exochitinase during growth of Streptomyces lividans pCHIO12 in soil microcosms amended with crab or fungal chitin. Appl Environ Microbiol 62, 1774-1780.

Wang, F., Xiao, X., Saito, A. \& Schrempf, H. (2002). Streptomyces olivaceoviridis possesses a phosphotransferase system that mediates specific, phosphoenolpyruvate-dependent uptake of $\mathrm{N}$-acetylglucosamine. Mol Genet Genomics 268, 344-351.

Xiao, X., Wang, F., Saito, A., Majka, J., Schlösser, A. \& Schrempf, H. (2002). The novel Streptomyces olivaceoviridis ABC transporter Ngc mediates uptake of $\mathrm{N}$-acetylglucosamine and $N, N^{\prime}$-diacetylchitobiose. Mol Genet Genomics 267, 429-439.

Zeltins, A. \& Schrempf, H. (1995). Visualization of $\alpha$-chitin with a specific chitin-binding protein (CHB1) from Streptomyces olivaceoviridis. Anal Biochem 231, 287-294.

Zeltins, A. \& Schrempf, H. (1997). Specific interaction of the Streptomyces chitin-binding protein CHB1 with $\alpha$-chitin: the role of individual tryptophan residues. Eur J Biochem 246, 557-564.

Zuber, S., Hynes, M. J. \& Andrianopoulos, A. (2002). G-protein signaling mediates asexual development at $25^{\circ} \mathrm{C}$ but has no effect on yeast-like growth at $37^{\circ} \mathrm{C}$ in the dimorphic fungus Penicillium mameffei. Eukaryot Cell 1, 440-447.

Edited by: C. W. Chen 